\title{
Hacia una Pedagogía Comprensiva - Restauradora
}

\author{
Towards a Comprehensive-Restorative Pedagogy \\ Hacia una Pedagogía Comprensiva - Restauradora
}

DOI: http://dx.doi.org/10.21803\%2Fpenamer.11.21.534

\author{
Luz Doris Chaparro \\ https://orcid.org/0000-0002-9747-2201 \\ Jenny Mercedes Aguirre \\ https://orcid.org/0000-0001-5116-4097
}

¿Cómo citar este artículo?

Chaparro, L. \& Aguirre, J. (2018). Hacia una Pedagogía Comprensiva - Restauradora. Pensamiento Americano, 11(21), 205-216

DOI: http://dx.doi.org/10.21803\%2Fpenamer.11.21.534

\section{Resumen}

El presente artículo es resultado del proceso de investigación de la fundamentación, consolidación, operacionalización e implementación de la Propuesta Pedagógica en la Corporación Universitaria Adventista (UNAC), de Medellín, Colombia. El objetivo de este proceso de investigación es orientar las acciones pedagógicos, curriculares, didácticos y evaluativos del quehacer universitario, fortalecer el proceso enseñanza- aprendizaje y responder a contextos emergentes y divergentes previamente delimitados, donde se promuevan y fortalezcan los cambios fundamentales en las prácticas educativas inherentesa susfundamentosfilosóficosque permiten la identidad institucional y sello distintivo. La metodología se define desde la investigación-acción, abordando las fases de diagnóstico, fundamentación e implementación desde el enfoque de investigación cualitativo, de carácter crítico descriptivo. Los resultados, además, de la consolidación y concreción de los principios y las acciones de lo estipulado en PEI, son los diferentes mecanismos y estrategias para la operacionalización o implementación de la Propuesta Pedagógica dentro del escenario de enseñanza aprendizaje. Se concluye, entonces, que la Propuesta Pedagógica, desde una visión comprensiva-restauradora, es un ejercicio reflexivo y de resignificación fundamentada en el accionar pedagógico, constituyéndose en un factor de primer orden para incrementar los niveles de pertinencia, calidad, efectividad y coherencia de todos los procesos adelantados por la UNAC.
\end{abstract}

PALABRAS CLAVE: Propuesta Pedagógica, proceso de enseñanzaaprendizaje comprensivo, operacionalización.

\begin{abstract}
This article is the result of the research process of the foundation, consolidation, operationalization and implementation of the Pedagogical Proposal in the Adventist University Corporation (UNAC) of Medellin, Colombia. The objective of this research process is to guide the pedagogical, curricular, didactic and evaluative actions of the task of the university, to strengthen the teaching-learning process and to respond to previously delimited emerging and divergent contests, where the fundamental changes in educational practices are promoted and strengthened inherent to its philosophical foundations that allow for institutional identity and distinctive stamp. The methodology is defined from the action research, approaching the phases of diagnosis, foundation and implementation from the qualitative research focus, of a descriptive critical nature. The results, in addition, of the consolidation and concretion of the principles and actions of the stipulated in PEI, are the different mechanisms and strategies for the operationalization or implementation of the Pedagogical Proposal within the teaching-learning scenario. It is concluded, then, that the Pedagogical Proposal, from a comprehensiverestorative vision, is a reflective and resignification exercise based on pedagogical action, becoming a factor of the first order to increase the levels of relevance, quality, effectiveness and coherence of all the processes advanced by the UNAC.
\end{abstract}

KEYWORDS: Pedagogical Proposal, comprehensive teachinglearning, operationalization. 


\section{Resumo}

O presente artigo é resultado do processo de pesquisa da fundamentação, consolidação, operacionalização e implementação da Proposta Pedagógica na Corporación Universitaria Adventista (UNAC), de Medellín, Colômbia. O objetivo deste processo de pesquisa é orientar as ações pedagógicas, curriculares, didáticas e avaliativas do fazer universitário, fortalecer o processo de ensino-aprendizagem e responder a contextos emergentes e divergentes previamente delimitados, onde se promovam e fortaleçam as mudanças fundamentais nas práticas educativas inerentes a seus fundamentos filosóficos que permitam a identidade institucional e selo distintivo.

A metodologia se define desde a pesquisa - ação, abordando as fases de diagnóstico, fundamentação e implementação desde o enfoque de pesquisa qualitativa, de caráter crítico descritiva. Os resultados, ademais, da consolidação e concretude dos princípios e das ações do estipulado no PEI são os diferentes mecanismos e estratégias para a operacionalização ou implementação da Proposta Pedagógica dentro do cenário de ensinoaprendizagem. Se conclui, então, que a Proposta Pedagógica, desde uma visão compreensiva-restauradora, é um exercício reflexivo e de ressignificação fundamentada no acionar pedagógico, que se constitui em um fator de primeira ordem para incrementar os níveis de pertinência, qualidade, efetividade e coerência de todos os processos adiantados pela UNAC.

PALAVRAS CHAVE: Proposta Pedagógica, processo de ensinoaprendizagem compreensivo, operacionalização.

\section{Perfil}

Mag. En Educación, Área Administración Educativa. Docente de tiempo completo e investigadora del grupo Pedagogía, Sociedad y Cultura de la Corporación Universitaria Adventista. Email: dchaparro@unac.edu.co

\section{Perfil}

Esp. En Docencia. Docente de tiempo completo e investigadora del grupo Pedagogía, Cultura y Sociedad de laCorporación Universitaria Adventista.

Email: jmaguirre@unac.edu.co

\section{Luz Doris Chaparro}

Licenciada en ciencias de la educación, ciencias sociales, económicas y políticas.

\section{Jenny Mercedes Aguirre}

Licenciada en pedagogía infantil 


\section{Introducción}

En la dinámica educativa en la cual nos vemos inmersos, los desafíos formativos que se gestionan y lideran deben responder a las diversas exigencias académicas contextuales, legales y conceptuales, que desde la sociedad del conocimiento se requieren, con sus respectivas demandas emergentes, como la apropiación de las Nuevas Tecnologías de la Información y la Comunicación, los efectos de fenómenos económicos y políticos, y la globalización y problemáticas socioculturales severas, entendidas como necesidades formativas relevantes que permitan una búsqueda de la dignificación del ser humano en una sociedad desfigurada. Cada una de estas exigencias debe ser considerada para atender los procesos de transformación universitaria como una necesidad de compromiso con la calidad, el mejoramiento y pertinencia educativa, realidad que no debe ser ajena a la UNAC.

La construcción de un modelo o propuesta pedagógica para una institución educativa implica la toma de decisiones con relación a la manera como esta debe conducirse. Es el marco de referencia permanente de las acciones que planifica y realiza la institución para mejorar la calidad de los procesos de aprendizaje-enseñanza, en un contexto específico, a fin de lograr cambios significativos de los educandos. Posibilita a la comunidad educativa definir colectivamente un conjunto de principios y acciones pedagógicas que concrecionan la intencionalidad del PEI para lograr su finalidad básica: La comprensión de la formación del ser humano, garantizando la coherencia de la práctica educativa dentro de la institución; proporcionar un marco orientador para lograr una actuación coordinada y eficaz de la comunidad educativa; responder con pertinencia a las necesidades de los agentes educativos; e ir generando una cultura organizacional a través de la filosofía educativa, los valores, los principios y la misión compartida.

Esta Propuesta Pedagógica se desarrolló por fases: diagnóstica, fundamentación e implementación; con acciones reflexivas, críticas y descriptivas para posibilitar la consolidación y la posterior implementación de la Propuesta Pedagógica desde una visión comprensiva-restauradora, y desarrollar el principio de la comprensión del ser humano en sus respectivas dimensiones, así como en su condición humana. La Propuesta Pedagógica sirve de referente para abordar los procesos educativos y formativos que emergen desde la reconstrucción de los saberes esenciales y desde la fundamentación de referentes filosóficos, psicológicos, axiológicos, sociológicos y pedagógicos, entre otros.

Por lo tanto, un modelo pedagógico plantea de forma integral un determinado fenómeno; desde el punto de vista teórico-práctico ofrece un marco de referencia para entender implicaciones, alcances, limitaciones y debilidades paradigmáticas que se dan para explicarlo (Gimeno \& Pérez, 1992).

Zubiría (2003) explica que los modelos pedagógicos y su comprensión son importantes en la medida en que posibilitan "las huellas o rastros que permiten reconstruir aspectos de la vida humana y que sirven de base para la reflexión y la investigación". Un modelo pedagógico es una amplia representación de un fenómeno, y a partir de él se pueden abrir espacios para la reflexión dentro del quehacer pedagógico. (pp. 8).

Entonces un modelo, en el caso de la pedagogía, no constituye una representación absoluta ni universal. Está formado a partir de diversos aportes teóricos, los cuales son tomados conforme a las necesidades que tienen los sujetos de aprendizaje, siendo modificables en cuanto a la perspectiva que se tenga de la 
pedagogía y los fines que se quiere alcanzar. Por lo tanto, un modelo pedagógico tiene en cuenta los lineamientos necesarios para promover el aprendizaje en un ambiente definido, con el fin de difundir y transmitir el conocimiento.

Desde la base anteriormente definida, los propósitos de la fundamentación e implementación, como operacionalización de la Propuesta Pedagógica, desde una visión comprensiva-restauradora, se centran en:

- Convertirse en un factor de primer orden en el empeño de incrementar los niveles de pertinencia, calidad, efectividad y coherencia de todos los procesos de enseñanza- aprendizaje adelantados por la Institución. Debe constituirse en la ruta de navegación para orientar los procesos pedagógicos, curriculares, didácticos y evaluativos del quehacer universitario.

- La necesidad de orientar la práctica pedagógica y la ejecución del currículo de manera articulada y fundamentada en teorías pedagógicas.

- La importancia y coherencia entre modelo educativo y el PEI, acordes con los pilares de la educación superior: Docencia, investigación, proyección social e internacionalización.

- La necesidad de introducir cambios en los procesos de enseñanza-aprendizaje y de implementar nuevas acciones curriculares, en correspondencia con los cambios, avances y tendencias científico-tecnológicas que respondan a las necesidades, retos y compromisos educativos emergentes.

- Delimitar nuevos roles del profesor y del estudiante, frente a los cambios, retosy demandas educativasy sociales, en un nuevo orden de relaciones educativas, científicasy tecnológicas.

\section{Metodología}

La presente investigación es de enfoque cualitativo, porque busca entender los planteamientos de la educación (filosóficos, axiológicos, legales, epistemológicos, entre otros); cada fase responde a un enfoque. En este orden de ideas, la investigación responde a su corte crítico.

El método de trabajo de la presente investigación tiene dos momentos. En el primer momento, se realiza la fundamentación teórica de la propuesta, con su estructura, enfoques y principios orientadores, respondiendo a la necesidad de orientar la práctica pedagógica y la ejecución del currículo de manera articulada y fundamentada en teorías pedagógicas; de introducir cambios en los procesos de enseñanza-aprendizaje y de implementar nuevas acciones curriculares, en correspondencia con los cambios, avances y tendencias científico-tecnológicas que respondan a las necesidades retos y compromisos educativos emergentes. Sierra (2012) considera que la reflexión sobre los modelos pedagógicos es un pretexto para replantear el quehacer pedagógico y la relación educación-pedagogía al dar cuenta de las prácticas escolares. Son ellas, las prácticas pedagógicas, las que muestran la realidad pedagógica que se establece entre maestros, estudiantes y cultura, como componentes del proceso educativo (pp. 23).

El segundo momento se denomina por Galeano (2004), gestión e implementación, pues, finalmente, se buscan espacios para la comunicación, socialización y divulgación de los resultados de la investigación, con el propósito de realimentar y validar la Propuesta Pedagógica sugerida.

Gutiérrez y Perafán (2002) afirman que la práctica pedagógica debe ser asumida como una construcción dialogante que 
permite diferentes autonomías en el saber, para la formación de educadores. Por ello es importante socializar el modelo pedagógico para que sea apropiado por la comunidad educativa.

La fase de operacionalización se aborda con base en la investigación-acción planteada por Carr y Kemmis (1986, citado en Aguilar \&Gómez Campillejo, 1991, pp. 298) en su modalidad emancipadora, comprendida como una herramienta para "el desarrollo curricular, promoción y desarrollo profesional, mejora de programas escolares a través de la identificación de las estrategias de acción y sometidas a la prácticas", y desde el diseño metodológico planteado por Escudero (1987), el cual se configura en un diagnóstico, acción e implementación del plan, observación, evaluación de la acción y reflexión sobre los resultados de evaluación.

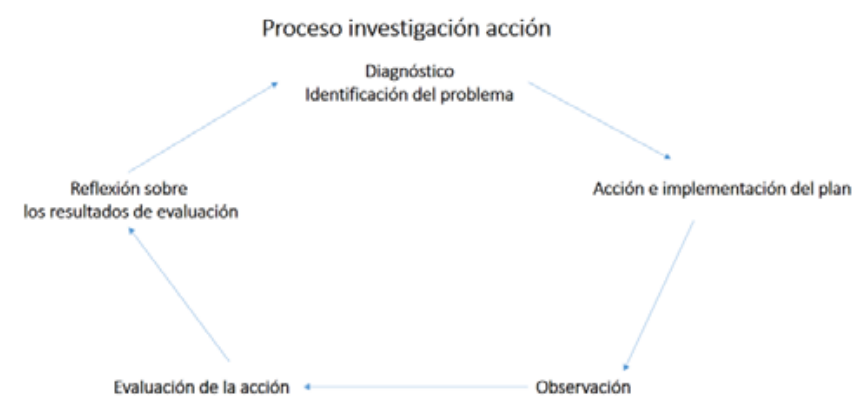

Figura 1: Proceso de investigación acción

Fuente: Elaboración propia, Jenny Aguirre, basada en el diseño metodológico de Escudero (1987).

Propuesta de investigación- acción a continuación, en forma detallada, en la figura 1 Para el diseño de los mecanismos se trabajó la investigación-acción, que parafraseando a Kemmis (1988, citado en González, Serpa, Gutiérrez \& Pirela, 2007) como una forma de indagación autorreflexiva desarrollada por participantes en situaciones sociales, en este caso a) las educacionales, con el objeto de mejorar la racionalidad y justicia de sus propias prácticas sociales o pedagógicas, b) su comprensión respecto de esas prácticas y c) las situaciones en las que se llevan a cabo (pp. 288)

La confiablidad y la validez, en la presente investigación, pretenden que la descomposición, interpretación y recomposición de la teoría sean claras y coherentes con la realidad. Se utiliza esencialmente como técnica de validación, la triangulación; se recoge información de varias teorías.

Para llevar a cabo todo el proceso de recolección de información fue necesario utilizar algunas estrategias metodológicas para cada una de fases, en aras al cumplimiento de los objetivos propuestos, que consisten en identificar los elementos característicos del proceso de indagación, consolidación, apropiación e implementación de la Propuesta Pedagógica, aplicados a una parte representativa de la comunidad educativa.

Se hicieron revisiones documentales y matrices de análisis; se aplicaron encuestas y se tuvo observación de prácticas educativas que permitieron acercarnos a la opinión, a sentir la percepción del grupo acerca de la Propuesta Pedagógica desde la pedagogía comprensiva- restauradora y la forma como se evidencia en el marco del proceso enseñanza-aprendizaje.

\section{Marco referencial}

Como se muestra en la Tabla 1, se propone entonces desarrollar esta investigación en fases, con sus respectivas etapas, para la construcción de la Propuesta Pedagógica: 
Tabla 1.

Propuesta Pedagógica. Fases y etapas

\begin{tabular}{|c|c|c|}
\hline Fase y etapas & Intencionalidad & Interrogantes \\
\hline $\begin{array}{l}\text { Diagnóstica } \\
\text { Etapa 1: Autorreflexiones y } \\
\text { discusiones dialécticas. } \\
\text { Etapa 2: Revisiones } \\
\text { documentales. } \\
\text { Etapa 3: Resignificación de } \\
\text { categorias desde potenciales } \\
\text { semánticos }\end{array}$ & $\begin{array}{l}\text { Carácter reflexivo, analítico } \\
\text { y hermenéutico, donde se } \\
\text { realizan revisiones } \\
\text { categoriales de un modelo } \\
\text { pedagógico. }\end{array}$ & $\begin{array}{l}\text { ¿Qué es un modelo } \\
\text { pedagógico? ¿Cuál es la } \\
\text { diferencia entre este y un } \\
\text { modelo educativo? } \\
\text { ¿Cuál es la pertinencia de un } \\
\text { modelo pedagógico y de una } \\
\text { propuesta pedagógica? } \\
\text { ¿Cuáles son las ventajas y } \\
\text { las desventajas de un } \\
\text { modelo pedagógico y de una } \\
\text { propuesta pedagógica? } \\
\text { ¿Cuáles son los constructos } \\
\text { o componentes de un } \\
\text { modelo pedagógico? }\end{array}$ \\
\hline $\begin{array}{l}\text { Fundamentación } \\
\text { Etapa 1: Estructuración y } \\
\text { formulación pedagógica. } \\
\text { Etapa 2: Consolidación. }\end{array}$ & 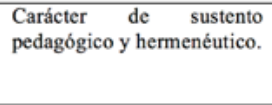 & $\begin{array}{l}\text { ¿Cuál debe ser la teoría o } \\
\text { apuesta pedagógica que } \\
\text { sustente a la Propuesta } \\
\text { Pedagógica de la UNAC? }\end{array}$ \\
\hline $\begin{array}{l}\text { Operacionalización } \\
\text { Etapa 1: Sensibilización y } \\
\text { promoción: Mecanismo de } \\
\text { comunicación } \\
\text { divulgación. } \\
\text { Etapa 2: Plan de acción: } \\
\text { Mecanismo de influencia } \\
\text { Etapa 3: Socialización y } \\
\text { capacitación: Mecanismo } \\
\text { de participación } \\
\text { Etapa 4: Seguimiento y } \\
\text { monitoreo: Mecanismo de } \\
\text { evaluación. }\end{array}$ & $\begin{array}{l}\text { Carácter pragmático o de } \\
\text { implementación. }\end{array}$ & $\begin{array}{l}\text { ¿Cuáles son las acciones, } \\
\text { mecanismos y estrategias } \\
\text { para la apropiación de la } \\
\text { Propuesta Pedagógica? } \\
\text { ¿Qué se requiere para la } \\
\text { implementación y cómo se } \\
\text { debe articular en los } \\
\text { procesos académicos y } \\
\text { curriculares de } \\
\text { Institución? }\end{array}$ \\
\hline
\end{tabular}

En la fase de fundamentación, el objetivo por considerar fue proponer una apuesta pedagógica que dinamice el proceso de enseñanza-aprendizaje.

La fundamentación pedagógica respondería a unos contextos emergentes y divergentes previamente delimitados, con el objeto de que a su vez se convierta en una estrategia e instrumento de investigación, donde se promuevan, fortalezcan y asuman los cambios fundamentales para todas las prácticas educativas que en la UNAC se desarrollen, desde una mirada introspectiva y crítica, y con referentes prospectivos articulados y estructurados desde lo epistemológico y lo filosófico, que permita ser coherente con la realidad y las intencionalidades formativas que se nos exigen.

La propuesta pedagógica, según Muñoz y Alvarado (2009), busca dar sustento pedagógico al quehacer educativo y formativo de una institución. Esta propuesta se asume de ma- nera plural y abierta, de allí que se permita la incorporación de otros discursos diferentes del propiamente pedagógico, para cumplir su cometido educativo y formativo. Se espera que pueda ser un punto de encuentro y diálogo con otros saberes, ciencias y disciplinas que se pueda requerir en un momento histórico y un contexto social y cultural propios de un proceso educativo dinámico.

La propuesta pedagógica ha de ser una noción esencial de las diversas prácticas con sus respectivas caracterizaciones, donde se integre, defina, jerarquice y optimice el conjunto de procesos pedagógicos y didácticos. Se requieren para la conformación de espacios de reflexión, de consolidación dialéctica, de estructuraciones epistemológicas, para que respondan a las aproximaciones de cada una de las demandas y exigencias de la dinámica educativa.

Según Echeverri y Zuluaga (2003), la propuesta pedagógica es concebida como "una manera abierta, dinámica y compleja de pensar las instituciones educativas, que permite empoderar al maestro atacando la raíz misma de la subalternidad intelectual, ese carácter de saber sometido, funcional de la tecnología educativa que ha sido asignado al saber pedagógico" (pp. 122).

Entendiéndose, entonces, la Propuesta Pedagógica como una construcción reflexiva que emerge de la capacidad de simbolización y representación de la tarea de enseñanza-aprendizaje, los protagonistas del proceso educativo de la UNAC deben considerarla para justificar y entender la amplitud de la práctica educadora, formativa y redentora; con la apropiación del conocimiento formalizado y las decisiones transformadoras que se requiere asumir.

La estructura, enfoques y componentes de la 
Propuesta se presentan a continuación en forma detallada en la figura 2.

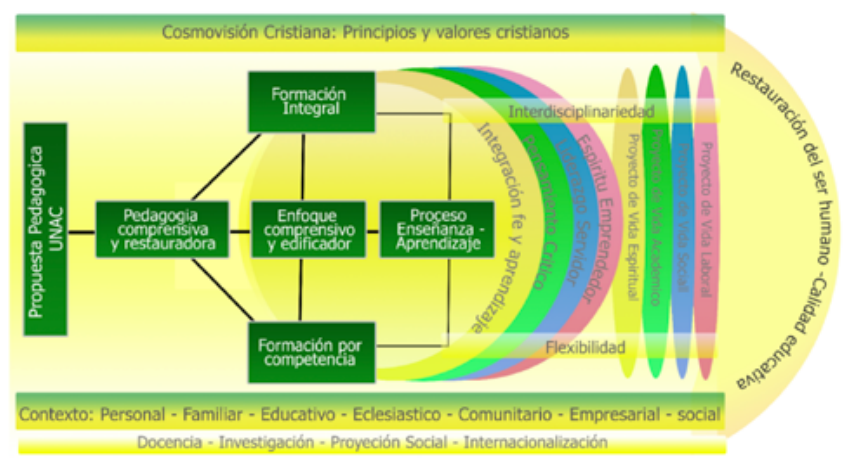

Figura 2. Enfoques y componentes de la propuesta.

Fuente: Elaboración propia de D. Chaparro y G. Padilla.

La Propuesta Pedagógica asumida por la UNAC, desde la pedagogía comprensiva- restauradora, se entiende como la posibilidad educativa y formativa de construcción permanente de un proceso de enseñanza-aprendizaje intencional, significativo y estructurado con la comprensión del conocer, del hacer y del ser. Implica la restauración de la condición humana en todas sus dimensiones, como factor determinante, y de nuestra perspectiva filosófica bíblico-cristiana: "Educar es redimir", que ante todo requiere la restauración en el hombre de la imagen de su Creador (Imago Dei), es decir, "devolverlo a la perfección con que había sido creado, promover el desarrollo del cuerpo, la mente y el alma, a fin de que se llevara a cabo el propósito divino de su creación, este es el objetivo de la educación, el gran propósito de la vida" (White, 1987. Pp. 16). Se caracteriza por la capacidad de apropiación y pertinencia para la resolución de problemas de la vida cotidiana, la toma de decisiones y el despliegue de la creatividad personal, con el fin de incidir en diferentes contextos y transformarlos.

En el libro "La Educación" encontramos que la educación y la redención se unen en su esfuerzo por restaurar al hombre a la plenitud de su ser original y a la armoniosa relación con Dios. Esta educación trabaja para que el educando se desarrolle físicamente, para que comprenda sus responsabilidades morales, para que se desarrolle como un ser personal, para que alcance la madurez de su individualidad en pleno ejercicio de sus facultades de pensar y actuar, para que se exprese con abnegación y amor, y para que su trabajo corresponda al de un sencillo servidor de su prójimo y de su Creador (Withe 1987, 16).

La Pedagogía Comprensiva-Restauradora exige:

- Restauración como edificación de la condición humana en todas sus dimensiones, y de los agentes educativos.

- La capacidad de apropiación, y pertinencia comprensiva y significativa del conocimiento.

- Capacidad para la resolución de problemas de la vida cotidiana.

• Enseñanza y aprendizajes comprensivos.

- Aplicación del proceso enseñanza-aprendizaje a diferentes contextos del accionar formativo.

Como se muestra en la Tabla 2, la relación que existe entre las dimensiones de la pedagogía comprensiva-restauradora es:

Tabla 2.

Relación entre las dimensiones de la pedagogía comprensiva-restauradora

\begin{tabular}{llll}
\hline DIMENSIÓN & COMPRENSIVA & RESTAURADORA \\
\hline Cognitiva & Comprensión cognitiva & Facultades mentales \\
\hline Discursiva comunicativa & Comprensión lectora-social & Facultades sociales \\
\hline Praxis & $\begin{array}{l}\text { Comprensión } \\
\text { psicopedagógica }\end{array}$ & Facultades para aprender \\
\hline Axiológica & $\begin{array}{l}\text { Comprensión ética } \\
\text { teleológica }\end{array}$ & y & Facultades espirituales \\
\hline Física & $\begin{array}{l}\text { Comprensión fisica } \\
\text { kinestésica }\end{array}$ & y & Facultades fisicas \\
\hline Fuente: Elaboración propia D Chaparro. & & \\
\hline
\end{tabular}

En esta línea, la pedagogía comprensiva-restauradora genera circunstancias para que la 
comprensión se potencie en ambientes de aprendizaje vivencial: propiciando los desempeños de los educandos en contextos de la realidad social, cultural, económica, familiar, personal y política, reflexionando de manera crítica y edificadora sobre el proceso, para hacer de esta apropiación un acto en favor de la comprensión sobre la restauración del ser humano en todas sus dimensiones.

Existen reflexiones sobre las prácticas restaurativas, basadas en la creencia de las habilidades que tienen las personas para realizar cambios positivos y reparar daños, todo esto basado en el establecimiento de la comunicación, donde la escucha es el objetivo principal para llegar a la comprensión del otro, antes que establecer las opiniones propias (Marín, 2017, pp. 216).

Lo anterior demuestra que la restauración y la comprensión se relacionan y se integran para el desarrollo de competencias de apropiación de conocimientos y aplicación de los mismos en la realidad del educando.

El proceso de enseñanza-aprendizaje comprensivo-restaurador está ligado a la acción, es decir, a la capacidad que tiene el educando de dominar los conocimientos y aplicarlos a otras situaciones. Es poder transferir esos conocimientos a contextos diferentes, es tener la posibilidad de explicarlos, mostrar sus hipótesis; es emplear el pensamiento.

Con tal fin es necesario tener en cuenta las siguientes premisas:

- ¿Cómo saber lo que se debe comprender y

restaurar en el proceso educativo?

- ¿Qué es lo que realmente se requiere para que los agentes educativos comprendan el proceso de enseñanza-aprendizaje?
- ¿Por qué y para qué es necesaria la comprensión en la restauración del ser humano?

- ¿Cómo enseñar y aprender comprensivamente?

La dimensión pedagógica se articula en un modelo educativo y se constituye en su dimensión fundamental. Es importante señalar que los fundamentos de la Propuesta Pedagógica de la UNAC recogen, por una parte, la manera como la Institución debe abordar el diseño curricular; y por otra, integran aproximaciones teóricas que permiten precisar el fundamento pedagógico y evidenciar más claramente los lineamientos que deben orientar su implementación.

En la etapa de la operacionalización, el objetivo fue establecer los mecanismos y estrategias para la apropiación e implementación de la Propuesta Pedagógica de la UNAC. Por lo tanto, los propósitos para la implementación y operacionalización de la Propuesta Pedagógica se centran en:

La oportunidad de trasladar el diseño pedagógico desde los diferentes niveles de concreción de la Propuesta Pedagógica a la práctica y a la ejecución del currículo de manera articulada.

La necesidad de introducir cambios en los procesos de enseñanza-aprendizaje y de implementar nuevas acciones curriculares, desde la implementación de las estrategias pedagógicas y didácticas que permitan la operacionalización de la Propuesta Pedagógica dentro del aula de clase.

Delimitar nuevos roles del profesor y del estudiante, frente a los cambios, retos y demandas educativas y sociales, en un nuevo orden de relaciones educativas, científicas y tecnológicas. 
Para la efectividad de la implementación de esta Propuesta Pedagógica en el ámbito educativo unacense se requiere que todos los agentes formadores se comprometan con los procesos de enseñanza-aprendizaje, desde los diferentes principios, fundamentos y concepciones planteados, que estos sean apropiados y articulados en cada una de las prácticas educativas, logrando concrecionar los ideales pedagógicos definidos en la comprensión y restauración del ser humano.

Por lo tanto, el proceso de operacionalización consiste en definir estrictamente variables en factores medibles, designando responsabilidades en los diferentes contextos y niveles de concreción del diseño, a la vez que se van caracterizando los procesos educativos ( $D^{\prime}$ Andreis, 2013). El modelo pedagógico de una institución es una estructura diseñada que encuentra su validez en la forma de operar, y en su comprensión, apropiación y asimilación por parte de la comunidad educativa. La teoría de Enrique Pichon-Rivière (Becerra, 2015) sobre el esquema conceptual y operativo sustenta que debe existir una estructuración de un determinado proceso, que consiste en aprenderlo, abordarlo, ordenarlo e interpretarlo. El equipo de trabajo solo logra aprender, en tanto, se opera lo planificado, la reflexión y la práctica sobre lo que se realiza puede llevar a un mejor aprendizaje.

En el proceso de operacionalización de la Propuesta Pedagógica se requiere la descripción de cada una de las acciones o medios, es decir, los mecanismos y estrategias necesarios para lograr la apropiación e implementación de la Propuesta por parte de la comunidad educativa, lo cual se hace posible solo a través de soluciones compartidas: Socialización, divulgación, comunicación, implementación, gestión, seguimiento y evaluación. El diseño de estos mecanismos como estrategias permitirá que se pueda dar sentido a un proceso de consolidación que ha tomado largo tiempo y esfuerzo de un equipo profesional que busca la verdadera formación del ser humano en todas dimensiones, en el quehacer educativo.

\section{Resultados}

Para la implementación de esta Propuesta Pedagógica en el ámbito educativo unacense se requiere que todos los agentes formadores se comprometan con los procesos de enseñanza-aprendizaje, desde los diferentes principios, fundamentos y concepciones planteados; que estos sean articulados en cada una de las prácticas educativas que se desarrollen en la Institución; contribuyan a la formación integral de los educandosy se evidencien en la calidad educativa requerida.

Ámbitos, niveles, acciones y estrategias para la concreción del diseño. Se muestran en la Tabla 3, los ámbitos, niveles, acciones y estrategias para la concreción del diseño.

Tabla 3.

Ámbitos, niveles, acciones y estrategias para la concreción del diseño.

\begin{tabular}{|c|c|c|c|c|}
\hline Ámbito & Niv & & Accion & Estrategias \\
\hline $\begin{array}{l}\text { Macro } \\
\text { contextual: } \\
\text { Diseño } \\
\text { curricular }\end{array}$ & $\begin{array}{l}\text { Vicerrectoría } \\
\text { Académica, } \\
\text { Oficina de } \\
\text { Curriculo, } \\
\text { facultades. }\end{array}$ & $\begin{array}{l}\text { Establecer } \\
\text { lineamientos, } \\
\text { criterios para } \\
\text { proceso } \\
\text { aprendizaje } \\
\text { esta Propuesta }\end{array}$ & \begin{tabular}{c}
\multicolumn{2}{|}{ los } \\
políticas y \\
articular el \\
enseñanza- \\
con base en \\
Pedagógica.
\end{tabular} & $\begin{array}{l}\text { Aprobar y promover esta } \\
\text { Propuesta Pedagógica con base } \\
\text { en sus respectivas concepciones, } \\
\text { principios y fundamentos. }\end{array}$ \\
\hline
\end{tabular}

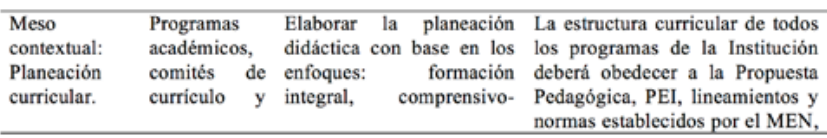

\begin{tabular}{|c|c|c|c|}
\hline & $\begin{array}{l}\text { jefes } \\
\text { áreas. }\end{array}$ & $\begin{array}{l}\text { edificador y formación } \\
\text { por competencias. }\end{array}$ & $\begin{array}{l}\text { para los programas de educación } \\
\text { superior y en las particularidades } \\
\text { de la disciplina respectiva. }\end{array}$ \\
\hline $\begin{array}{l}\text { Micro } \\
\text { contextual: } \\
\text { Secuencias } \\
\text { didácticas }\end{array}$ & Docentes & $\begin{array}{l}\text { Aplicar los principios, } \\
\text { componentes, niveles y } \\
\text { actividades de la } \\
\text { enseñanza-comprensiva- } \\
\text { restauradora, asi como las } \\
\text { orientaciones didácticas en } \\
\text { las respectivas prácticas } \\
\text { educativas. }\end{array}$ & $\begin{array}{l}\text { Generen experiencias de } \\
\text { enseñanza-aprendizaje, como } \\
\text { realización personal y profesional } \\
\text { de manera significativa, } \\
\text { comprensiva y restauradora. }\end{array}$ \\
\hline
\end{tabular}


En la Tabla 4 se muestran las etapas de operacionalización de la Propuesta Pedagógica:

Tabla 4.

Etapas de operacionalización. Fase: Operacionalización de la propuesta pedagógica

\begin{tabular}{lll}
\hline ETAPA & MECANISMOS & \multicolumn{1}{c}{ ESTRATEGIAS } \\
\hline $\begin{array}{l}\text { Sensibilización } \\
\text { y promoción }\end{array}$ & $\begin{array}{l}\text { Comunicación } \\
\text { divulgación }\end{array}$ & $\begin{array}{l}\text { Divulgación de los lineamientos de la Propuesta } \\
\text { Pedagógica }\end{array}$ \\
\hline Plan de acción & De influencia & $\begin{array}{l}\text { Diseño de estrategias de gestión. } \\
\text { Formulación de estrategias metodológicas y } \\
\text { didácticas para los diferentes componentes de la } \\
\text { Propuesta. }\end{array}$ \\
\hline $\begin{array}{l}\text { Generación de insumos y recursos pedagógicos de } \\
\text { las estrategias. }\end{array}$ \\
\hline capacitación & Participación & $\begin{array}{l}\text { Espacios de reflexión y participación. } \\
\text { Apropiación de las estrategias, politicas y } \\
\text { mecanismos por parte de los agentes educativos. }\end{array}$ \\
& & $\begin{array}{l}\text { Actualización y formación permanente de la } \\
\text { comunidad educativa. }\end{array}$ \\
\hline $\begin{array}{l}\text { Seguimiento } \\
\text { monitoreo }\end{array}$ & Evaluación & $\begin{array}{l}\text { Caracterización de las prácticas de enseñanza y } \\
\text { aprendizaje. } \\
\text { Revisión y análisis del quehacer docente en su } \\
\text { contexto pedagógico. } \\
\text { Diseño de matriz de indicadores de gestión para } \\
\text { determinar la apropiación e implementación de la } \\
\text { PP. }\end{array}$ \\
\hline
\end{tabular}

Fuente: Elaboración propia, D. Chaparro y J. Aguirre.

Se muestran en la Tabla 5 las estrategias de operacionalización de la Propuesta Pedagógica de la UNAC.

Tabla 5 .

Estrategias de operacionalización de la Propuesta.

\begin{tabular}{|c|c|}
\hline Estrategias & Acciones \\
\hline GESTIÓN & $\begin{array}{l}\text { Establecer los lineamientos, politicas y criterios para } \\
\text { articular el proceso enseñanza-aprendizaje. } \\
\text { Elaborar la planeación didáctica con base en los enfoques; } \\
\text { formación integral y formación por competencias. } \\
\text { Aplicar los principios y desarrollar los componentes, asi } \\
\text { como las orientaciones didácticas en las respectivas } \\
\text { prácticas educativas. }\end{array}$ \\
\hline $\begin{array}{l}\text { METODOLÓGICAS } \\
\text { PROCESO DE ENSEÑANZA- } \\
\text { APRENDIZAJE COMPRENSIVO }\end{array}$ & $\begin{array}{l}\text { Condiciones: intencionalidades formativas. } \\
\text { Componentes: hilos conductores, metas, desempeños de } \\
\text { comprensión. } \\
\text { Actividades de la comprensión: explicación, } \\
\text { ejemplificación, aplicación, justificación, comparación, } \\
\text { contextualización y generalización. } \\
\text { Según Perkins \& Blythe (2005). }\end{array}$ \\
\hline DIDÁCTICAS & $\begin{array}{l}\text { Ejes integradores: pensamiento crítico, fe y aprendizaje, } \\
\text { espiritu emprendedor, liderazzo servidor. } \\
\text { Dispositivos didácticos: habilidades de pensamiento. } \\
\text { Reflexiones y reseñas críticas. } \\
\text { Relatorias. } \\
\text { Protocolos y portafolios. } \\
\text { Proyectos de vida: espiritual, académico, laboral y social. } \\
\text { Proyectos: de vida e integradores. }\end{array}$ \\
\hline
\end{tabular}

Se muestran en la Tabla 6, los diferentes mecanismos por implementar en la Propuesta Pedagógica de la UNAC.

Tabla 6.

Mecanismos para la implementación de la Propuesta.

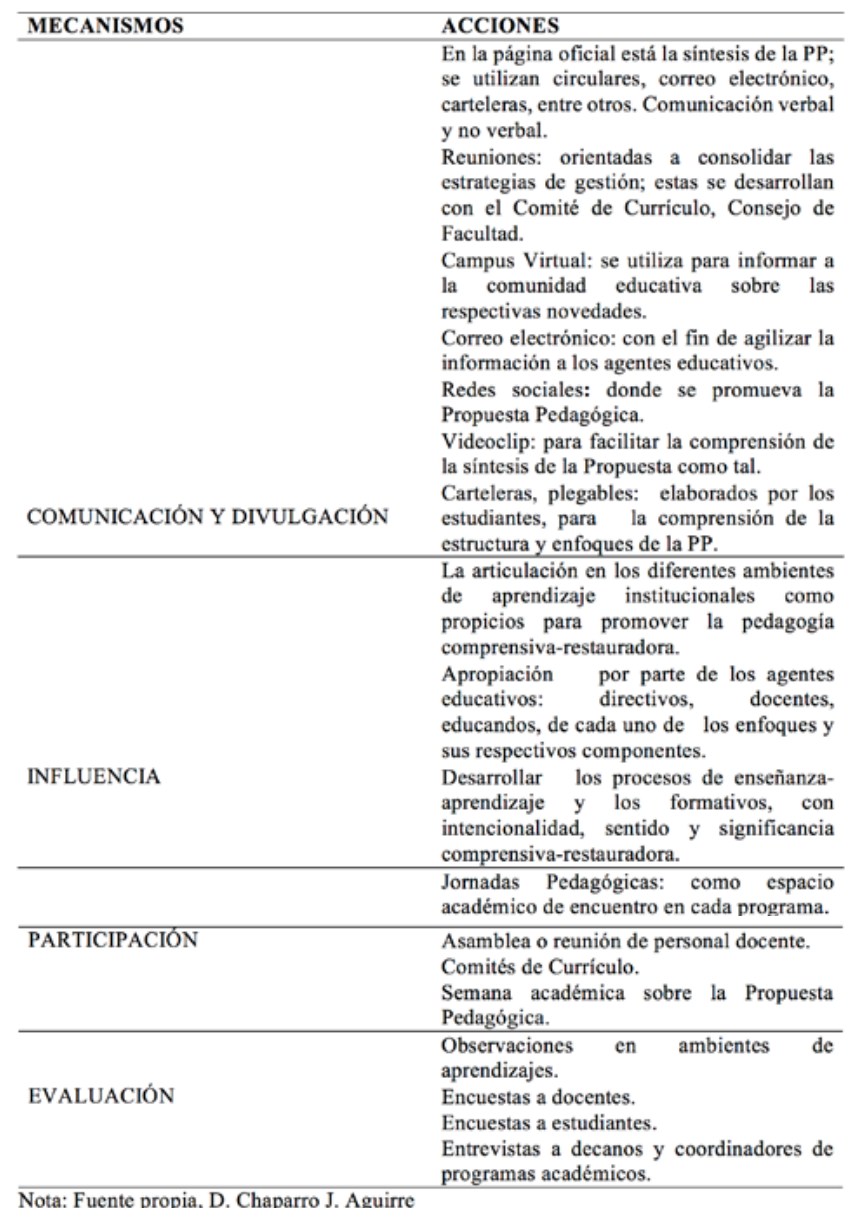

Nota: Fuente propia, D. Chaparro J. Aguirre

\section{(n)}




\section{Conclusiones}

Dado el dinamismo de la Institución, la Propuesta Pedagógica es perfectible, lo que indica que es necesaria la autorreflexión crítica permanente en las diferentes instancias de participación, y en las exigencias del quehacer educativo.

La Propuesta Pedagógica ha de ser una noción esencial de las diversas prácticas con sus respectivas caracterizaciones, donde se integre, defina, jerarquice y optimice el conjunto de hechos de los diferentes procesos pedagógicos y didácticos que se requieren para la consolidación de espacios de reflexión, consolidación dialéctica y estructuraciones epistemológicas, para que respondan a las aproximaciones de cada una de las demandas y exigencias de la dinámica educativa.

Para la comprensión de esta Propuesta Pedagógica, como operacionalización en el contexto universitario, ha de ser participativa, así como su implementación, seguimiento y evaluación, son referente esencial e indispensable para el análisis de la calidad de los procesos académicos y curriculares (Cordero, 2013).

El sustento teórico de la Propuesta Pedagógica de la UNAC es coherente y aplicable con los enfoques y filosofía de la UNAC, lo cual genera en los estudiantes una experiencia en el aprendizaje comprensivo. Esta construcción reflexiva y sistemática posibilita a quienes aprenden, a involucrarse y comprometerse con su propio proceso de comprensión y de mejoramiento continuo.

La diferencia específica entre la pedagogía comprensiva-restauradora y otros enfoques, modelos o constructos de la educación y la formación reside en que aquella promueve un mecanismo para alcanzar propósitos de formación integral, desarrollo de las dimensiones en el educando y de su condición hu- mana, de manera restauradora, a la par que da la aproximación a un proceso de enseñanza-aprendizaje comprensivo.

La Propuesta Pedagógica de la UNAC favorece el sentido de la comprensión en la vida personal, profesional y laboral de cada individuo para que tenga una experiencia académica profesional, tecnológica o técnica.

Para la implementación de la Propuesta Pedagógica en al ámbito educativo unacense se requiere que todos los agentes formadores se comprometan con los procesos de enseñanza- aprendizaje, desde los diferentes principios, fundamentos y concepciones planteados; que estos sean articulados en cada una de las prácticas educativas, y que contribuyan a la formación de integral de los educandos, lo cual debe evidenciarse en la calidad educativa requerida.

El proceso enseñanza-aprendizaje comprensivo restaurador se fundamenta en el principio esencial del aprender haciendo, preparando no solamente para el aprendizaje, sino para la vida. 


\section{Referencias}

Aguilar, P. F., \& Gómez Capillejo, M. A. (1991). Aproximación Teórica a la Investigación Acción y su Proyección Práctica en la Realidad Educativa. Revista universitaria de formación del profesorado (10), 295309. Recuperado el 23 de 04 de 2018, de https:// dialnet.unirioja.es/descarga/articulo/117750.pdf

Becerra, G. (2015). Riviére, Enrique Pichón: Los Orígenes de la Psicología Social Argentina. RELMECS, 5(1). Recuperado el 07 de 09 de 2017, de biblioteca. universia.net/...pichon-riviere-origenes-psicologiasocial.../62200128.html

Cordero, I. (2013). Didáctica y enseñanza del Derecho en las universidades. Pensamiento Americano. 6(11) 29-34

D'Andreis Zapata, A. (2013). Responsabilidad social empresarial RSE: un estudio desde sus teorías, Precursores y críticos. Ad-gnosis, 2(2), 49 - 64.

Echeverri, J., \& Zuluaga, O. (2003). Campo intelectual de a educación y campo pedagógico. En pedagogía y epistemología., 111-123.

Escudero, J. (1987). La investigación- acción en el panorama actual de la investigación educativa: algunas tendencias. Revista de Innovación e Investigación Educativa., 14-25.

González, N; Serpa, M, L; Gutiérrez D y Pirela, C. (2007). La investigación educativa en el hacer docente. Caracas. Universidad Pedagógica Experimental Libertador. Laurus, vol. 13, núm. 23, 2007, pp. 279309. Artículo disponible en http://www.redalyc.org/ articulo.oa?id=76102315

Gutiérrez, E. F. R. \& Perafán, E. L. (2002). Currículo y práctica pedagógica. Popayán: Taller Editorial Universidad del Cauca.

Gimeno, J. \& Pérez, Á. (1992). Comprender y transformar la enseñanza. Madrid: Morata.

Galeano M, M. (2004b). Estrategias de investigación social cualitativa. El giro en la mirada. Medellín: La Carreta Editores.

Marín, C. G. (2017). Las prácticas restaurativas en la creación de espacios de paz dentro de la escuela. Pensamiento Americano, 11(20), 213-226. Recuperado el 10 de Julio de 2018, de http://www. coruniamericana.edu.co/publicaciones/ojs/index. php/pensamientoamericano/article/view/511/pdf_61

Muñoz, G. \& Alvarado, D. (2009). La integralidad como multidimensionalidad: un acercamiento desde la teoría crítica. Hologaramática, 103-116.

Perkins, D., \& Blythe, T. (2005). Ante todo, la comprensión. Revista Magisterio Educación y Pedagogía., Vol. 14, abril.

Sierra, C. M. (2012). La Práctica Docente desde los Modelos Pedagógicos. En C. M. Sierra, Diplomado en Pedagogía Universitaria (pág. 23). Medellín: Uniremington. Recuperado el $\mathrm{O} 2$ de $\mathrm{O} 2$ de 2018, de https://es.calameo.com/ read/005436481bd9e4c9ddcc2

White, E. G. (1987). La Educación. Florida: Asociación Publicadora Interamericana.

Zubiría, J. d. (2003). Tratado de Pedagogía Conceptual: Los modelos pedagógicos. 8.uni

2018, Vol. 11(21) 205-216 @ The Author(s) 2018 Reprints and permission: www.americana.edu.co

https://www.coruniamericana.edu.co/publicaciones/ojs/index.php/pensamientoamericano/index 